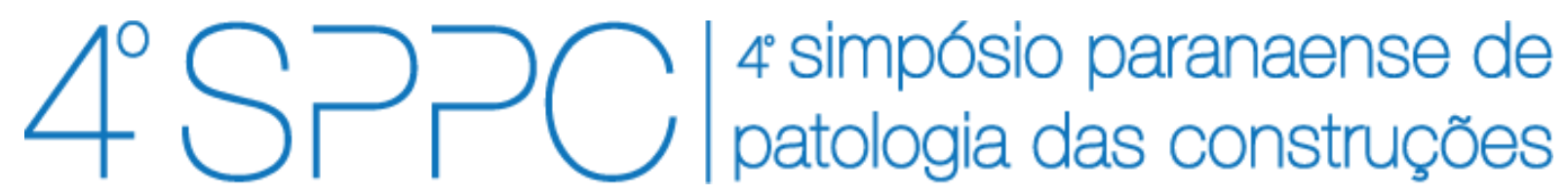

ISSN 2526-7248 artigo n. 4SPPC105, pp. 38-47, 2019

\title{
Ensaios acelerados de penetração de cloretos aplicados à estimativa do coeficiente de difusão do concreto e à vida útil de estruturas em ambiente marinho
}

\author{
Wally, Gustavo Bosel ${ }^{1}$; Sell Júnior, Félix Krolow2; Magalhães, Fábio Costa ${ }^{3}$ \\ ${ }^{1}$ Mestrando em Engenharia Oceânica, Universidade Federal do Rio Grande - \\ FURG, gustavo.wally@gmail.com \\ ${ }^{2}$ Mestrando em Engenharia Oceânica, Universidade Federal do Rio Grande - \\ FURG, felixsell@gmail.com \\ ${ }^{3}$ Professor Doutor em Engenharia Civil, Instituto Federal do Rio Grande do Sul - \\ Campus Rio Grande, fabiocmagalhaes@gmail.com
}

Resumo: A durabilidade e a vida útil das estruturas em concreto armado são atualmente uma preocupação generalizada entre os envolvidos com a construção civil. A corrosão das armaduras causada pela ação de cloretos tem recebido atenção especial visto que há elevada concentração desses íons em zonas costeiras. Neste trabalho são avaliados os coeficientes de difusão de três dosagens de concreto utilizando o método prescrito pela NT Build 492 e também através de uma relação com a carga passante determinada pela ASTM C 1202. É também realizada uma avaliação da correlação dos resultados de ambos os métodos. Por fim é determinado o cobrimento da armadura necessário ao atendimento de diferentes vidas úteis de serviço. Os resultados mostram que existem divergências entre os resultados apresentados por ambos os métodos e que essas podem ser influenciadas pela qualidade do concreto avaliado.

Palavras-chave: Penetração de cloretos, Previsão de vida útil, Corrosão.

Abstract: The durability and the service life of reinforced concrete structures are currently a widespread concern among those involved in construction. The corrosion of the reinforcement caused by the action of chlorides has received special attention since there is high concentration of this ions in coastal zones. In this work the diffusion coefficients of three concrete admixtures are evaluated using the method prescribed by NT Build 492 and also through a relation from the through load determined by ASTM C 1202. A correlation evaluation of the results of both methods is presented. Finally, it is determined the covering of the rebar required to attend different service lifetimes. The results show that there are differences between the results presented by both methods and that these can be influenced by the quality of the concrete.

Keywords: Chloride penetration, Service life prediction, Corrosion. 
WALLY, G. B.; SELL JUNIOR, F. K.; MAGALHÃES, F. C., ENSAIOS DE MIGRAÇÃO DE CLORETOS APLICADOS À ESTIMATIVA DO COEFICIENTE DE DIFUSÃO DO CONCRETO E DA VIDA ÚTIL DE ESTRUTURAS EM AMBIENTE MARINHO. $4^{\circ}$ Simpósio

Paranaense de Patologia das Construções (40 SPPC), artigo 4SPPC105, pp. 38 - 47, 2019. DOI: 10.4322/2526-7248.027

\section{Introdução}

Aspectos relacionados à durabilidade e à vida útil de estruturas em concreto armado ganharam importância crescente na concepção de projetos estruturais nos últimos anos devido ao desempenho inadequado de muitas estruturas quanto à durabilidade. A penetração de cloretos e a carbonatação são as principais causas da deterioração precoce de estruturas em concreto armado [1-2].

As considerações acerca da durabilidade e vida útil das estruturas na fase de projeto são recentes, sendo introduzidas na Europa e nos Estados Unidos na década de 1990 e no Brasil em 2003, após a publicação da NBR 6118 (posteriormente atualizada em 2014). Mais tarde, em 2013, foi reiterada pela publicação da NBR 15575, a qual considera a durabilidade das estruturas de concreto como parâmetro de desempenho das construções civis [3].

Yu et al. [4] afirmam que o ambiente marinho é o mais agressivo às estruturas de concreto pois a água do mar, além de aumentar a probabilidade de corrosão da armadura, pode causar danos ao concreto, possibilitando a ocorrência de processos físicos e químicos simultaneamente, como ataque por cloretos, sulfatos, erosão superficial e outros. Gjørv [5] corrobora ao afirmar que a alta penetração de cloretos nas estruturas e a corrosão prematura das armaduras são os grandes problemas enfrentados nos projetos de durabilidade e na operação e manutenção de estruturas em concreto armado em ambientes de severa agressividade.

Segundo Ollivier e Torrenti [6], quanto maior for a dificuldade imposta ao ingresso de agentes nocivos na estrutura de poros do concreto, mais durável este será. $\mathrm{O}$ fenômeno que governa o ingresso de cloretos em estruturas de concreto em ambientes marinhos é a difusão [7] e a difusividade do concreto pode ser tomada como um indicador da qualidade do mesmo, estando a ela associados o tipo de aglomerante, relação água/cimento, uso de aditivos químicos e outros fatores.

Devido à longa duração de ensaios de difusão no concreto, ensaios de migração, nos quais o fluxo iônico ocorre devido à imposição de uma diferença de potencial elétrico, têm sido utilizados na avaliação da qualidade do concreto e na determinação da difusividade do mesmo de forma acelerada [8].

Nesse contexto, no presente trabalho é realizada uma avaliação do coeficiente de difusão aparente e da vida útil de serviço de três traços de concreto produzidos com cimento pozolânico, a fim de avaliar a qualidade dos concretos frente a ambientes agressivos bem como analisar a difusividade calculada por meio de dois ensaios de migração e suas implicações na estimativa de vida útil e no cobrimento requerido à armadura.

\section{Metodologia}

\subsection{Produção dos concretos}

Foram produzidos três traços de concreto, todos contendo cimento Portland CP IV32 da marca Votoran, brita $n^{-} 1$ e areia média quartzosa oriundas da cidade de Pelotas/RS. A dosagem seguiu a metodologia apresentada pelo IPT/EPUSP [9]. 
WALLY, G. B.; SELL JUNIOR, F. K.; MAGALHÃES, F. C., ENSAIOS DE MIGRAÇÃO DE CLORETOS APLICADOS À ESTIMATIVA DO COEFICIENTE DE DIFUSÃO DO CONCRETO E DA VIDA ÚTIL DE ESTRUTURAS EM AMBIENTE MARINHO. $4^{\circ}$ Simpósio

Paranaense de Patologia das Construções (40 SPPC), artigo 4SPPC105, pp. 38 - 47, 2019. DOI: 10.4322/2526-7248.027

O teor ideal de argamassa seca $(\alpha)$ foi definido em $53 \%$. O resumo das dosagens é apresentado na Tabela 1.

Tabela 1: Dosagens dos concretos utilizados.

\begin{tabular}{cccc}
\hline Parâmetro & Traço Rico & $\begin{array}{c}\text { Traço } \\
\text { Intermediário }\end{array}$ & $\begin{array}{c}\text { Traço } \\
\text { Pobre }\end{array}$ \\
\hline Traço unitário (1:m) & $1: 3,5$ & $1: 4,7$ & $1: 6,2$ \\
Relação a/c & 0,46 & 0,53 & 0,65 \\
Teor de aditivo (\%) & 0,5 & 0,5 & 0,5 \\
Abatimento $(\mathrm{mm})$ & 60 & 80 & 110 \\
\hline
\end{tabular}

A moldagem e a cura dos corpos de prova foram realizadas conforme indicado pela NBR 5738 [10]. Foram moldados corpos de prova com dimensões $\phi 100 \times 200 \mathrm{~mm} e$ estes passaram por cura submersa até a idade de 28 dias, na qual deu-se início à realização dos ensaios propostos. Os corpos de prova foram cortados em fatias de $50 \mathrm{~mm}$, como preconizado pelas normas utilizadas, e cada cilindro teve suas laterais impermeabilizadas para garantir um fluxo iônico unidirecional.

\subsection{Determinação do coeficiente de difusão aparente}

O coeficiente de difusão aparente foi determinado utilizando o método normatizado pela NT Build 492 [11] e através da carga passante medida pelo ensaio prescrito pela ASTM C 1202 [12]. Após a determinação dos coeficientes de difusão aparentes, os concretos foram classificados quanto à penetrabilidade de cloretos conforme apresentado na Tabela 2.

Tabela 2: Resistência à penetração de cloretos [13].

\begin{tabular}{|c|c|}
\hline $\begin{array}{l}\text { Coeficiente de difusão aparente } \\
\qquad\left(10^{-8} \mathrm{~cm}^{2} / \mathrm{s}\right)\end{array}$ & $\begin{array}{l}\text { Penetrabilidade de } \\
\text { cloretos }\end{array}$ \\
\hline & Alta \\
\hline $10-15$ & Moderada \\
\hline $5-10$ & Baixa \\
\hline $2,5-5$ & Muito baixa \\
\hline$<2,5$ & Desprezível \\
\hline
\end{tabular}

\subsubsection{NT Build 492}

O ensaio apresentado pela NT Build 492 [11] determina o coeficiente de difusão aparente em estado não-estacionário $\left(D_{n s s m}\right)$ do concreto. A configuração do ensaio é apresentada na Fig. 1.

As amostras foram submetidas inicialmente a uma tensão de $30 \mathrm{~V}$, a qual foi ajustada de acordo com a corrente inicial, podendo variar entre 10 e $60 \mathrm{~V}$, conforme a qualidade do concreto, seguindo a prescrição da norma. Ao final do teste os concretos foram rompidos diametralmente e neles foi pulverizada solução de $\mathrm{AgNO}_{3}$ a $0,1 \mathrm{M}$. Assim é possível verificar até qual profundidade ocorreu a penetração de cloretos. A Fig. 2 apresenta a fase inicial de realização do ensaio. 


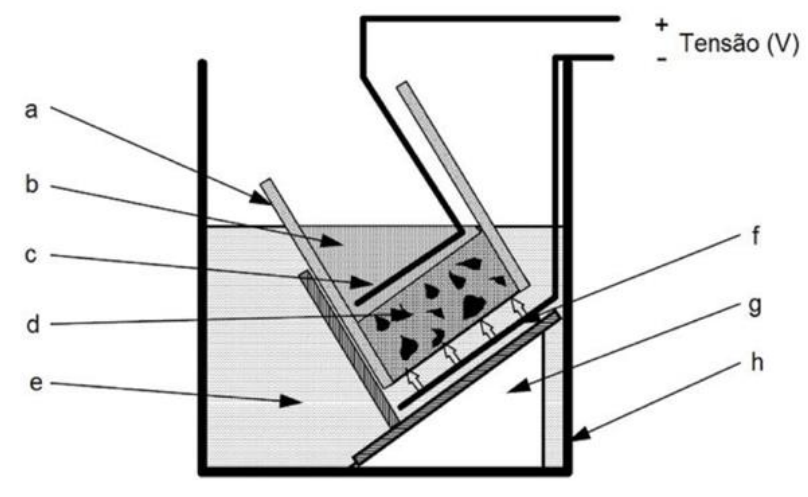

Figura 1: Esquema da realização do ensaio prescrito pela NT Build 492 [14].

onde (a) proteção de borracha; (b) solução contendo $\mathrm{NaOH}$; (c) ânodo; (d) amostra de concreto; (e) solução contendo $\mathrm{NaCl}$; (f) cátodo; (g) suporte plástico e (h) caixa plástica.

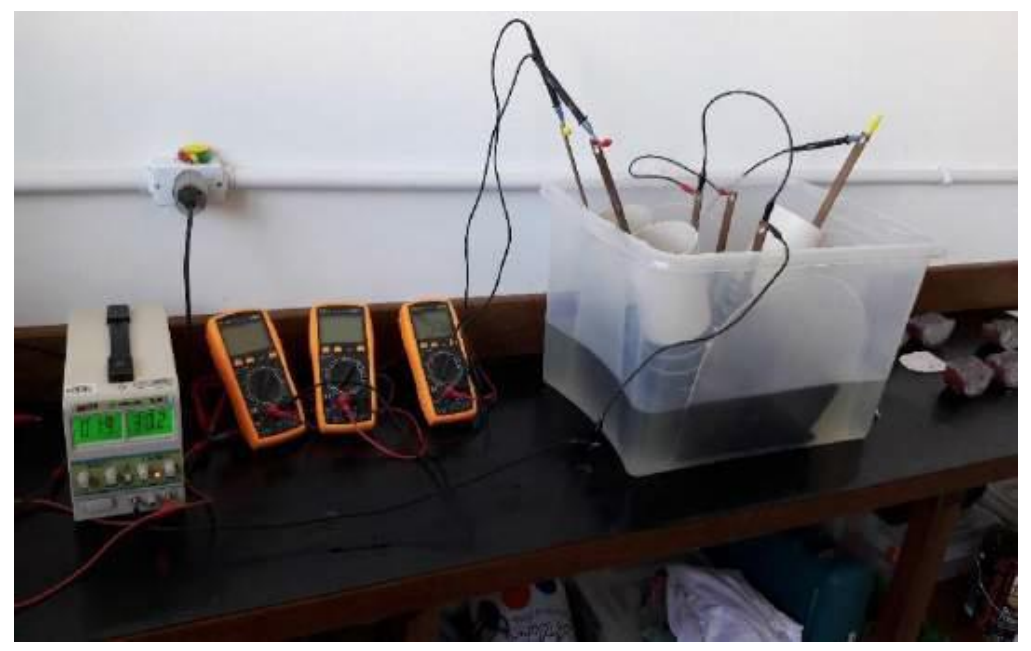

Figura 2: Realização do ensaio prescrito pela NT Build 492.

Por fim, o coeficiente de difusão aparente ( $\left.D_{\text {nssm }}\right)$ foi determinado através da Eq. 1.

$$
D_{\text {nssm }}=\frac{0,0239(273+T) L}{(U-2) t}\left(x_{D}-0,0238 \sqrt{\frac{(273+T) L x_{D}}{U-2}}\right)
$$

Na Eq. $1, D_{n s s m}$ é o coeficiente de difusão aparente $\left(\times 10^{-8}\right)\left[\mathrm{cm}^{2} / \mathrm{s}\right], T$ é o valor médio entre as temperaturas inicial e final $\left[{ }^{\circ} \mathrm{C}\right], L$ é a espessura da amostra [mm], $U$ é o valor absoluto da voltagem aplicada $[\mathrm{V}], x_{D}$ é a média das profundidades de penetração [mm] e $t$ é o tempo de duração do ensaio [h]. 
WALLY, G. B.; SELL JUNIOR, F. K.; MAGALHÃES, F. C., ENSAIOS DE MIGRAÇÃO DE CLORETOS APLICADOS À ESTIMATIVA DO COEFICIENTE DE DIFUSÃO DO CONCRETO E DA VIDA ÚTIL DE ESTRUTURAS EM AMBIENTE MARINHO. $4^{\circ}$ Simpósio

Paranaense de Patologia das Construções (40 SPPC), artigo 4SPPC105, pp. 38 - 47, 2019. DOI: 10.4322/2526-7248.027

\subsubsection{ASTM C 1202}

Foi realizado ensaio de resistência à penetração de cloretos conforme a ASTM C 1202 [12], o qual consiste no monitoramento da corrente elétrica passante através do corpo de prova de concreto. Após preparação, saturação e impermeabilização dos corpos de prova, as amostras foram confinadas entre dois compartimentos, um deles preenchido com solução de $\mathrm{NaCl}$ a $3 \%$ e o outro com $\mathrm{NaOH}$ a $0,3 \mathrm{~N}$. O polo negativo da fonte foi ligado à face que continha a solução de $\mathrm{NaCl}$ e, por consequência, o polo positivo foi ligado à extremidade com $\mathrm{NaOH}$.

Uma das grandes críticas impostas a este método é o fato de que a tensão aplicada $(60 \mathrm{~V})$ é extremamente elevada, acarretando em superaquecimento do sistema e consequentes prejuízos à confiabilidade da medição [15]. Assim, optou-se por aplicar uma tensão de menor intensidade $(30 \mathrm{~V})$ e posteriormente fez-se a correção dos valores de corrente medidos no circuito elétrico através da Lei de Ohm. A Fig. 3 e a Fig. 4 apresentam, respectivamente, o esquema de realização do ensaio e 0 aparato utilizado para a realização do mesmo

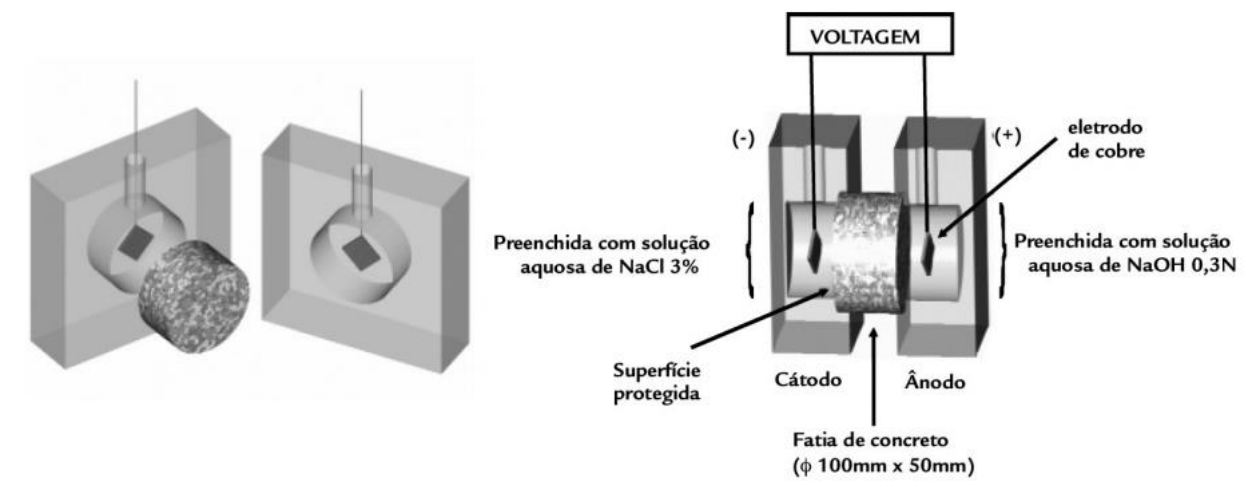

Figura 3: Esquema da realização do ensaio prescrito pela ASTM C 1202 [16].

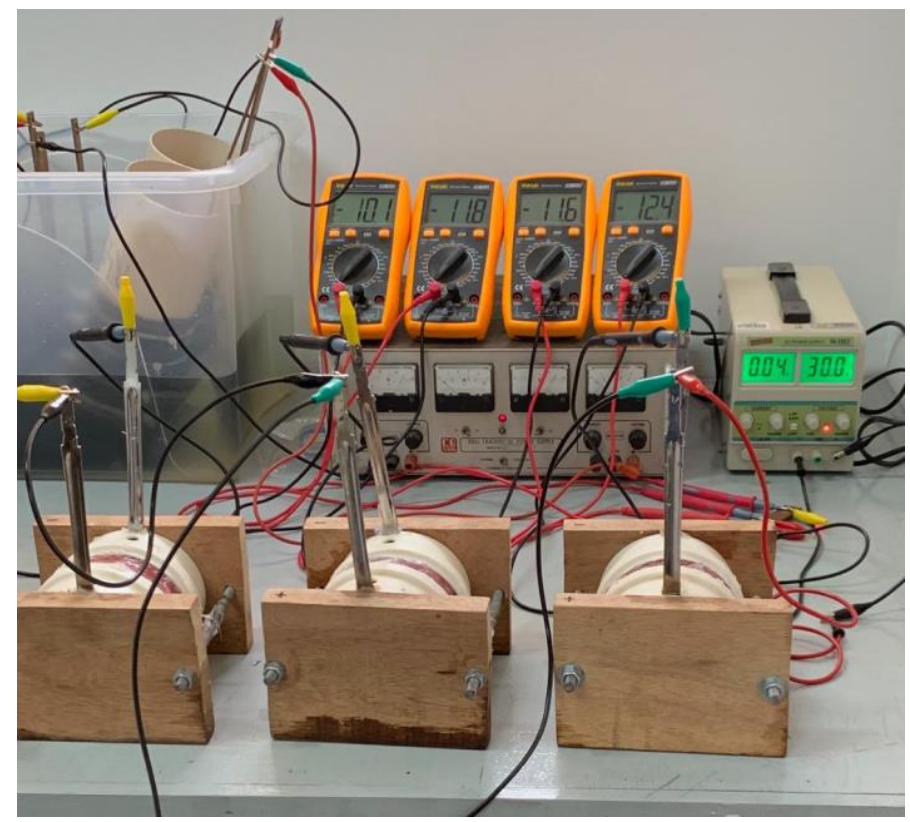

Figura 4: Equipamento utilizado no ensaio de resistência à penetração de cloretos. 
WALLY, G. B.; SELL JUNIOR, F. K.; MAGALHÃES, F. C., ENSAIOS DE MIGRAÇÃO DE CLORETOS APLICADOS À ESTIMATIVA DO COEFICIENTE DE DIFUSÃO DO CONCRETO E DA VIDA ÚTIL DE ESTRUTURAS EM AMBIENTE MARINHO. $4^{\circ}$ Simpósio

Paranaense de Patologia das Construções (40 SPPC), artigo 4SPPC105, pp. 38 - 47, 2019. DOI: 10.4322/2526-7248.027

Durante as 6 horas de duração do ensaio são realizadas leituras da corrente passante a cada 30 minutos. Ao fim do ensaio a carga passante total é calculada conforme a Eq. 2.

$$
Q=900\left(I_{0}+2 I_{30}+2 I_{60}+\ldots+2 I_{330}+I_{360}\right)
$$

onde $Q$ é a carga passante [C], $I_{0}$ é a corrente medida imediatamente após a aplicação da tensão $[\mathrm{A}]$ e $I_{t}$ é a corrente medida $t$ minutos após o início do ensaio $[\mathrm{A}]$.

Com vistas à avaliação do coeficiente de difusão dos concretos, o método apresentado por Berke e Hicks [17], o qual relaciona a carga passante determinada conforme a Eq. 2 com o coeficiente de difusão aparente do concreto $\left(\mathrm{C}_{\mathrm{da}}\right)$, foi utilizado. Trata-se de uma metodologia não normatizada, porém empregada por vários autores [17-18]. Esta é apresentada na Eq. 3.

$$
C_{d a}=\left(0,0103 \times 10^{-8}\right) Q^{0,84}
$$

Na Eq. 3, $C_{d a}$ é o coeficiente de difusão aparente $\left[\mathrm{cm}^{2} / \mathrm{s}\right]$ e $Q$ é a carga total passante [C] (calculada conforme a Eq. 2).

\subsection{Previsão de vida útil}

A estimativa da profundidade da penetração de cloretos para as diversas idades estudadas foi realizada utilizando a solução da segunda lei da difusão de Fick, apresentada na Eq. 4 e na Eq. 5, a qual tem sido utilizada por diversos autores [16, $19,20]$.

$$
\begin{aligned}
& P C=2(z) \sqrt{D t} \\
& \operatorname{erf}(z)=1-\frac{C_{c l}-C_{0}}{C_{S}-C_{0}}
\end{aligned}
$$

Em que $D$ é o coeficiente de difusão do concreto $\left[\mathrm{cm}^{2} / \mathrm{s}\right], t$ é o tempo de vida útil [anos], erf(z) é a função erro de Gauss, $P C$ (penetração de cloretos) é a profundidade em que a concentração de cloretos é capaz de despassivar a armadura [cm], Co é a concentração inicial de cloretos [\%], Cs é a concentração superficial de cloretos [\%] e $C_{c l}$ é a concentração de cloretos em função da profundidade e do tempo [\%].

Foi adotada a concentração superficial $\left(\mathrm{C}_{\mathrm{S}}\right)$ igual a 2,07 \%, valor apresentado por Guimarães [21] para uma zona totalmente de névoa em ambiente marinho na cidade de Rio Grande/RS. Utilizou-se o teor de 0,4 \% como concentração crítica de cloretos. Os coeficientes de difusão empregados foram os medidos experimentalmente utilizando a metodologia descrita na seção 2.2. 
WALLY, G. B.; SELL JUNIOR, F. K.; MAGALHÃES, F. C., ENSAIOS DE MIGRAÇÃO DE CLORETOS APLICADOS À ESTIMATIVA DO COEFICIENTE DE DIFUSÃO DO CONCRETO E DA VIDA ÚTIL DE ESTRUTURAS EM AMBIENTE MARINHO. $4^{\circ}$ Simpósio

Paranaense de Patologia das Construções (40 SPPC), artigo 4SPPC105, pp. 38 - 47, 2019. DOI: 10.4322/2526-7248.027

\section{Resultados e discussões}

A carga passante e os coeficientes de difusão calculados para cada um dos traços, bem como a classificação dos concretos quanto à resistência à penetração de cloretos [13], são apresentados na Tabela 3.

Tabela 3: Resultados dos ensaios realizados.

\begin{tabular}{ccccc}
\hline Parâmetro & & Traço 1:3,5 & Traço 1:4,7 & Traço 1:6,2 \\
\hline Carga passante Q [C] & & 2574 & 3726 & 4410 \\
$\mathrm{C}_{\text {da }}\left[\mathrm{cm}^{2} / \mathrm{s}\right]$ & Valor & $7,55 \mathrm{E}-08$ & $1,03 \mathrm{E}-07$ & $1,18 \mathrm{E}-07$ \\
& Classificação & Baixa & Moderada & Moderada \\
$\mathrm{D}_{\text {nssm }}\left[\mathrm{cm}^{2} / \mathrm{s}\right]$ & Valor & $8,56 \mathrm{E}-08$ & $1,01 \mathrm{E}-07$ & $2,38 \mathrm{E}-07$ \\
& Classificação & Baixa & Moderada & Alta \\
\hline
\end{tabular}

Com base na Tabela 2, observa-se que o traço 1:3,5 é o que apresenta melhor resistência à penetração de cloretos, uma vez que as cargas passantes nos concretos com dosagem 1:4,7 e 1:6,2 são, respectivamente, $44,75 \%$ e $71,33 \%$ superiores ao traço 1:3,5. Tal fato se justifica visto que esta é também a ordem crescente das relações água/cimento $(\mathrm{a} / \mathrm{c})$ utilizadas. Quanto menor a relação a/c, mais densa é a matriz cimentícia e menor é a ligação entre os poros através dos quais ingressam os íons.

Nota-se também que ocorrem variações entre os valores calculados de $C_{d a}$ e $D_{n s s m}$. A Fig. 5 apresenta a relação entre os coeficientes determinados $\left(C_{d a} / D_{n s s m}\right)$ para cada uma das dosagens avaliadas.

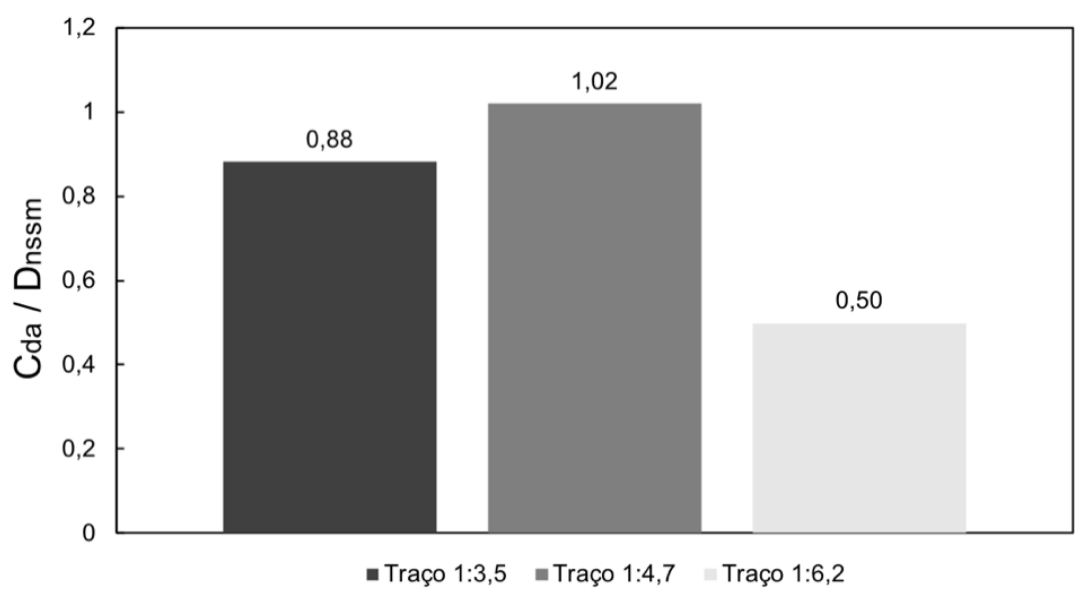

Figura 5: Relação entre os coeficientes de difusão obtidos a partir das duas metodologias utilizadas.

Percebe-se, a partir da Fig. 5, que os métodos apresentaram boa aproximação de resultados para as dosagens 1:3,5 e 1:4,7. No entanto, para o traço 1:6,2, o valor determinado a partir da Eq. 3 corresponde à metade do valor de $D_{\text {nssm. }}$. Deve-se atentar a esse fato visto que esses valores são indicadores da durabilidade potencial dos concretos e influenciam na estimativa da vida útil da estrutura e/ou no cobrimento requerido à armadura. 
WALLY, G. B.; SELL JUNIOR, F. K.; MAGALHÃES, F. C., ENSAIOS DE MIGRAÇÃO DE CLORETOS APLICADOS À ESTIMATIVA DO COEFICIENTE DE DIFUSÃO DO CONCRETO E DA VIDA ÚTIL DE ESTRUTURAS EM AMBIENTE MARINHO. $4^{\circ}$ Simpósio

Paranaense de Patologia das Construções (40 SPPC), artigo 4SPPC105, pp. 38 - 47, 2019. DOI: 10.4322/2526-7248.027

$\mathrm{Na}$ Tabela 4 é apresentado como as variações na determinação dos coeficientes de difusão, evidenciadas na Fig. 5, implicam na necessidade de cobrimento da armadura para que a estrutura cumpra uma determinada vida útil. Para tal, utilizouse a relação entre o cobrimento necessário a partir dos valores medidos para $C_{d a} \mathrm{e}$ $D_{n s s m}\left(X_{C d a} / X_{D n s s m}\right)$.

Tabela 4: Variações no cobrimento da armadura necessário em função do método utilizado na determinação do coeficiente de difusão.

\begin{tabular}{ccc}
\hline $\begin{array}{c}\text { Vida útil de projeto } \\
\text { (anos) }\end{array}$ & Concreto avaliado & $\boldsymbol{X}_{\text {Cda }} / \boldsymbol{X}_{\text {Dnssm }}$ \\
\hline \multirow{2}{*}{5} & $1: 3,5$ & 0,94 \\
& $1: 4,7$ & 1,01 \\
& $1: 6,2$ & 0,71 \\
\hline & $1: 3,5$ & 0,94 \\
& $1: 4,7$ & 1,01 \\
& $1: 6,2$ & 0,71 \\
\hline & $1: 3,5$ & 0,94 \\
& $1: 4,7$ & 1,01 \\
& $1: 6,2$ & 0,71 \\
\hline & $1: 3,5$ & 0,94 \\
75 & $1: 4,7$ & 1,01 \\
& $1: 6,2$ & 0,71 \\
\hline$-0-0.3,5$ & 0,94 \\
100 & $1: 3,4$ & 1,01 \\
& $1: 4,7$ & 0,71 \\
\hline
\end{tabular}

Com base na Tabela 4 verifica-se a influência do método de determinação do coeficiente de difusão do concreto na estimativa de sua vida útil. Nota-se, especialmente, que a variação de $50 \%$ entre os valores de coeficiente de difusão aparente obtidos para o traço 1:6,2 $\left(C_{d a} / D_{n s s m}=0,5\right)$ implicou em uma variação significativa no cobrimento da armadura necessário para que se cumpra uma determinada vida útil de projeto $\left(X_{C d a} / X_{\text {Dnssm }}=0,71\right)$. Tal fato corrobora a necessidade de uma normatização nacional para avaliação de indicadores de durabilidade do concreto, como o coeficiente de difusão, uma vez que os diversos métodos disponíveis na literatura podem apresentar valores distintos e que implicam em previsões de vida útil significativamente diferentes.

Percebe-se, ainda, que o aumento da vida útil de projeto não gerou alterações na relação $X_{C d a} / X_{D n s s m}$ de cada concreto. Este fato ocorre pois se trata de uma avaliação determinística na qual somente a vida útil de projeto foi alterada, não sendo consideradas, por exemplo, as variabilidades que cada parâmetro de entrada do modelo pode assumir, bem como o fator de envelhecimento do concreto, o qual tende a reduzir a difusividade do material ao passar do tempo. 
WALLY, G. B.; SELL JUNIOR, F. K.; MAGALHÃES, F. C., ENSAIOS DE MIGRAÇÃO DE CLORETOS APLICADOS À ESTIMATIVA DO COEFICIENTE DE DIFUSÃO DO CONCRETO E DA VIDA ÚTIL DE ESTRUTURAS EM AMBIENTE MARINHO. $4^{\circ}$ Simpósio

Paranaense de Patologia das Construções (40 SPPC), artigo 4SPPC105, pp. 38 - 47, 2019. DOI: 10.4322/2526-7248.027

\section{Conclusões}

Com base nos resultados apresentados, conclui-se que:

- Existem divergências entre os coeficientes obtidos por meio das duas metodologias utilizadas, principalmente quando avaliado o concreto com menor relação a/c, e que tais divergências implicam também na estimativa de vida útil das estruturas;

- A vida útil de projeto não interfere na relação entre os cobrimentos de armadura necessários determinados a partir das duas metodologias de determinação do coeficiente de difusão $\left(X_{C d a} / X_{D n s s m}\right)$. No entanto, esse comportamento mostrou-se sensível às dosagens dos concretos avaliados.

\section{Agradecimentos}

O presente trabalho foi realizado com apoio da Coordenação de Aperfeiçoamento de Pessoal de Nível Superior - Brasil (CAPES) - Código de Financiamento 001.

\section{Referências}

[1] Beushausen, H.; Torrent, R.; Alexander, M. G. (2019) Performance-based approaches for concrete durability: State of the art and future research needs, Cement and Concrete Research, 119:11-20.

[2] Beushausen, H. (2019) Introduction. In: Performance-Based Specifications and Control of Concrete Durability - State-of-the-Art Report, RILEM TC 230-PSC. New York, USA.

[3] Magalhães, F. C. (2018) Proposição de um modelo para a penetração de íons cloreto em concreto: estudo dos parâmetros intervenientes e análise probabilística. Tese (Doutorado), Universidade Federal do Rio Grande do Sul UFRGS, Porto Alegre, Brasil.

[4] Yu, B.; Ning, C.; Li, B. (2017) Probabilistic durability assessment of concrete structures in marine environments: reliability and sensitivity analysis, China Ocean Engineering, 31:63-73.

[5] Gjørv, O. E. (2015) Projeto de durabilidade de estruturas de concreto em ambientes de severa agressividade. São Paulo, São Paulo, Brasil.

[6] Ollivier, J. P.; Torrenti, J. M. (2014) A estrutura porosa dos concretos e as propriedades de transporte. In: Durabilidade do concreto: Bases científicas para a formulação de concretos duráveis de acordo com o ambiente. São Paulo, São Paulo, Brasil.

[7] Justnes, H.; Kim, M. O.; Ng, S.; Qian, X. (2016) Methodology of calculating required chloride diffusion coefficient for intended service life as function of concrete cover in reinforced marine structures, Cement and Concrete Research, 73:316-323. 
WALLY, G. B.; SELL JUNIOR, F. K.; MAGALHÃES, F. C., ENSAIOS DE MIGRAÇÃO DE CLORETOS APLICADOS À ESTIMATIVA DO COEFICIENTE DE DIFUSÃO DO CONCRETO E DA VIDA ÚTIL DE ESTRUTURAS EM AMBIENTE MARINHO. $4^{\circ}$ Simpósio

Paranaense de Patologia das Construções (40 SPPC), artigo 4SPPC105, pp. 38 - 47, 2019. DOI: 10.4322/2526-7248.027

[8] Tong, L.; Gjørv, O. E. (2001) Chloride diffusivity based on migration testing, Cement and Concrete Research, 31:973-982.

[9] Helene, P.; Terzian, P. (1993) Manual de Dosagem e Controle do Concreto. São Paulo, São Paulo, Brasil.

[10]NBR 5738 (2016) Concreto - Procedimento para moldagem e cura de corpos de prova. Associação Brasileira de Normas Técnicas - ABNT, Rio de Janeiro, Brasil.

[11] NT Build 492 (1999) Concrete, mortar and cement-based repair materials: chloride migration coefficient from non-steady-state migration experiments. NORDTEST, Espoo, Finland.

[12] ASTM C 1202 (2012) Standard Test Method for Electrical Indication of Concrete's Ability to Resist Chloride Ion Penetration. American Society for Testing and Materials - ASTM, West Conshohocken, USA.

[13] Nilsson, L.; Ngo, M. H.; Gjørv, O. (1998) High-performance repair materials for concrete structures in the port of Gothenburg. In: Second International Conference on Concrete Under Severe Conditions: Environment and Loading, 2:1193-1198.

[14] Martins, A. M. (2016) Transporte de cloretos em concretos com adições minerais e o desempenho em relação à corrosão das armaduras. Dissertação (Mestrado), Universidade Federal de Goiás - UFG, Goiânia, Brasil.

[15] Andrade, C. (1993) Calculation of chloride diffusion coefficients in concrete from ionic migration measurements, Cement and Concrete Research, 23:724742.

[16] Medeiros, M. H. F.; Gobbi, A.; Groenwold, J. A.; Helene, P. (2013) Previsão da vida útil de concreto armado de alta resistência com adição de metacaulim e sílica ativa em ambientes marinhos, Revista Escola de Minas, 66:59-65.

[17] Berke, N.; Hicks, M. (1992) Estimating the Life Cycle of Reinforced Concrete Decks and Marine Piles Using Laboratory Diffusion and Corrosion Data. In: Corrosion Forms and Control for Infrastructure, West Conshohocken, US.

[18] Andrade, C.; Whiting, D. (1996) A comparison of chloride ion diffusion coefficients derived from concentration gradients and non-steady state accelerated ionic migration, Materials and Structures, 29:476-484.

[19] Helene, P.; Guignone, G.; Vieira, G.; Roncetti, L.; Moroni, F. (2018) Avaliação da penetração de cloretos e da vida útil de concretos autocicatrizantes ativados por aditivo cristalino, Revista IBRACON de Estruturas e Materiais, 11:544-563.

[20] Ribeiro, D. V. (2018) Estrutura de poros e mecanismos de transporte no concreto. In: Corrosão e Degradação em Estruturas de Concreto - Teoria, controle e técnicas de análise e intervenção, Rio de Janeiro, Rio de Janeiro, Brasil.

[21] Guimarães, A. T. C. (2000) Vida útil de estruturas de concreto armado em ambientes marítimos. Tese (Doutorado), Universidade de São Paulo - USP, São Paulo, Brasil. 